\title{
Rationality of Dempster-Shafer combination step by step
}

\author{
CHEN qiang, LIU Wanshun, WAN Bojun, WANG Haifeng \\ (School of Electrical Engineering and Automation, Jiang Xi University of Science and Technology, \\ Gan Zhou, 341000, China)
}

Key words: step-by-step combination algorithm, Dempster-Shafer evidence combination, conflicting evidence, convergence.

Abstract: In this paper, provided a solution that evidence are sequentially used in pair aiming at the problem of multi-evidence combination. Several theorems and deductions are presented and proved. The step-by-step combination algorithm mixing the classic Dempster-Shafer evidence combination method and the weighted average method is proposed for combination of severe conflicting evidence. The proposed algorithm has better convergence effectiveness and lesser uncertainty than the algorithms proposed in reference literature.

\section{Introduction}

To use Dempster-Shafer evidence fusion method, the problem of conflict evidence combination need to be solved firstly. The improved schemes in general can be divided into two categories: combination rule modifying method and evidence modifying method. Yager proposed an improvement measure in which the normalization factor is cancelled, and conflicting information is assigned to uncertain item [1]. The improved rules proposed by Sun, Deng, Li, Xiao et al realize in general conflict management through calculating the mutual support level between the evidence or the evidence distance[2-5]. Murphy proposed an evidence average combination rule[6]. Lefevre proposed a combination rule which allow an arbitrary or adapted assignment of the conflicting mass to subsets [7]. Ali proposed an evidence modifying rule that the belief of the evidence are modified according to joint probability of two events[8]. But these improved have shortcomings. Such as the calculating process is not simple and direct enough, or the convergence of the combining results is not very satisfying. In this study, We propose a simple and direct conflicting evidence combination algorithm which is a step-by-step combination algorithm mixing the classic Dempster-Shafer evidence combination method and the weighted average method. In addition, Step by step combination method is widely used by the researchers. But the rationality of this method in theory is less involved in literature so far. We study the rationality of the step by step combination method. Several important properties of the combination method be presented and proved.

\section{Dempster-Shafer theory}

Provided that space $X: X=\left\{x_{1}, x_{2}, \ldots x_{n}\right\}$ is constituted by mutual exclusive and exhaustive elements, called frame of discernment. The power set of $X 2^{x}$ is a proposition set, defined function $m: 2^{x} \rightarrow[0,1]$, if any proposition of the set $A\left(A \in 2^{x}\right)$ satisfies:

$$
\left\{\begin{array}{c}
m(\phi)=0 \\
\sum_{A \subseteq X} m(A)=1
\end{array}\right.
$$

Where, $m$ is called basic probability assignment(BPA) of $A$. If $m(A)>0$, then $A$ is called a focal 
element[9]. For $n$ independent evidence $m_{1}(*), m_{2}(*), \cdots m_{n}(*)$,Dempster-Shafer evidence theory provides the following evidence combination formula:

$$
\begin{aligned}
& m(A)=\frac{1}{1-K} \sum_{A_{i} \cap B_{j} \cap C_{k} \cap \ldots=A} m_{1}\left(A_{i}\right) m_{2}\left(B_{j}\right) m_{3}\left(C_{k}\right) \ldots \\
& K=\sum_{A_{i} \cap B_{j} \cap C_{k} \cap .=\varnothing} m_{1}\left(A_{i}\right) m_{2}\left(B_{j}\right) m_{3}\left(C_{k}\right) \ldots
\end{aligned}
$$

The value of $K$ in formula (2) or (3) indicates the degree of evidence conflict each other. The prerequisite of evidence combination is $0<K<1$.

\section{Normalization and astringency of the continuous substeps method for Dempster-Shafer evidence combination}

Several theorems and deductions which relate to the continuous Dempster-Shafer evidence combination are proposed in this section, and the proofs are provided.

Theorem 1: Provided a frame of discernment $X, A$ is a focal element of $X$. In $X$, there are $N$ nonvoid subsets of $A$ : $A_{1}, A_{2}, \ldots A_{\mathrm{N}}$, these subsets do not overlap each other, and there are $n$ rows of independent evidence:

$\left\{\begin{array}{c}\left.m_{1} *^{*}\right): m_{1}\left(A_{1}\right), m_{1}\left(A_{2}\right), \ldots m_{1}\left(A_{N}\right) \\ m_{2}(*): m_{2}\left(A_{1}\right), m_{2}\left(A_{2}\right), \ldots m_{2}\left(A_{N}\right) \\ \ldots \ldots \\ m_{n}(*): m_{n}\left(A_{1}\right), m_{n}\left(A_{2}\right), \ldots m_{n}\left(A_{N}\right)\end{array}\right.$

Suppose the evidence satisfy the normalization condition that:

$\sum_{j=1}^{N} m_{i}\left(A_{j}\right)=1, i=1,2, \ldots n$

If the evidence of (4) are combined in pair sequentially using the combination formulas of the classical Dempster-Shafer evidence theory, then the last combined results must be:

$$
m\left(A_{j}\right)=\frac{\prod_{i=1}^{n} m_{i}\left(A_{j}\right)}{\sum_{j=1}^{N} \prod_{i=1}^{n} m_{i}\left(A_{j}\right)},(j=1,2, \ldots N)
$$

Proof: We obtain the combination result of the foremost 2 rows of evidence (the number of evidence groups is $k=2$ ), according to (3), the degree of conflict is:

$$
\begin{aligned}
& K_{1}=m_{1}\left(A_{1}\right) \sum_{j=2}^{N} m_{2}\left(A_{j}\right)+m_{1}\left(A_{2}\right) \sum_{j \neq 2} m_{2}\left(A_{j}\right)+\ldots+m_{1}\left(A_{n}\right) \sum_{j=1}^{N-1} m_{2}\left(A_{j}\right) \\
& 1-K_{1}=1-\left[m_{1}\left(A_{1}\right) \sum_{j=2}^{N} m_{2}\left(A_{j}\right)+m_{1}\left(A_{2}\right) \sum_{j \neq 2} m_{2}\left(A_{j}\right)+\ldots+m_{1}\left(A_{n}\right) \sum_{j=1}^{N-1} m_{2}\left(A_{j}\right)\right] \\
& \text { for } \sum_{j=1}^{N} m_{i}\left(A_{j}\right)=1, i=1,2, \ldots n \text { therefore: } \\
& 1-K_{1}=\sum_{j=1}^{N} m_{1}\left(A_{j}\right) m_{2}\left(A_{j}\right)
\end{aligned}
$$


According to (2), the combined results of step1 is:

$$
\left\{\begin{array}{r}
m^{1}\left(A_{1}\right)=\frac{m_{1}\left(A_{1}\right) m_{2}\left(A_{1}\right)}{\sum_{j=1}^{N} m_{1}\left(A_{j}\right) m_{2}\left(A_{j}\right)} \\
m^{1}\left(A_{1}\right)=\frac{m_{1}\left(A_{2}\right) m_{2}\left(A_{2}\right)}{\sum_{j=1}^{N} m_{1}\left(A_{j}\right) m_{2}\left(A_{j}\right)} \\
\ldots \ldots \ldots . \\
m^{1}\left(A_{1}\right)=\frac{m_{1}\left(A_{N}\right) m_{2}\left(A_{N}\right)}{\sum_{j=1}^{N} m_{1}\left(A_{j}\right) m_{2}\left(A_{j}\right)}
\end{array}\right.
$$

It can be written as:

$$
m^{1}\left(A_{j}\right)=\frac{\prod_{i=1}^{2} m_{i}\left(A_{j}\right)}{\sum_{j=1}^{N} \prod_{i=1}^{2} m_{i}\left(A_{j}\right)},(j=1,2, \ldots N)
$$

Now, we suppose that the combination result of the foremost $k$ rows of evidence is:

$$
m^{k-1}\left(A_{j}\right)=\frac{\prod_{i=1}^{k} m_{i}\left(A_{j}\right)}{\sum_{j=1}^{N} \prod_{i=1}^{k} m_{i}\left(A_{j}\right)},(j=1,2, \ldots N)
$$

Then we can obtain the result of combining (8) with the next row of evidence in (4) as follows. The degree of conflict is:

$$
\begin{gathered}
K_{k}=\frac{\prod_{i=1}^{k} m_{i}\left(A_{1}\right) \sum_{j=2}^{N} m_{k+1}\left(A_{j}\right)+\prod_{i=1}^{k} m_{i}\left(A_{2}\right) \sum_{j \neq 2} m_{k+1}\left(A_{j}\right)+\ldots+\prod_{i=1}^{k} m_{i}\left(A_{N}\right) \sum_{j=1}^{N-1} m_{k+1}\left(A_{j}\right)}{\sum_{j=1}^{N} \prod_{i=1}^{k} m_{i}\left(A_{j}\right)} \\
1-K_{k}=\frac{\sum_{j=1}^{N} \prod_{i=1}^{k} m_{i}\left(A_{j}\right)-\prod_{i=1}^{k} m_{i}\left(A_{1}\right) \sum_{j=2}^{N} m_{k+1}\left(A_{j}\right)-\prod_{i=1}^{k} m_{i}\left(A_{2}\right) \sum_{j \neq 2} m_{k+1}\left(A_{j}\right)-\ldots-\prod_{i=1}^{k} m_{i}\left(A_{N}\right) \sum_{j=1}^{N-1} m_{k+1}\left(A_{j}\right)}{\sum_{j=1}^{N} \prod_{i=1}^{k} m_{i}\left(A_{j}\right)}
\end{gathered}
$$

for $\sum_{j=1}^{N} m_{i}\left(A_{j}\right)=1, i=1,2, \ldots n$ therefore:

$$
1-K_{k}=\frac{\sum_{j=1}^{N} \prod_{i=1}^{K+1} m_{i}\left(A_{j}\right)}{\sum_{j=1}^{N} \prod_{i=1}^{k} m_{i}\left(A_{j}\right)}
$$

The combined results of the foremost $K+1$ rows of evidence is: 


$$
\left\{\begin{aligned}
& m^{k}\left(A_{1}\right)= \frac{\prod_{i=1}^{K+1} m_{i}\left(A_{1}\right)}{\sum_{j=1}^{N} \prod_{i=1}^{K+1} m_{i}\left(A_{j}\right)} \\
& m^{k}\left(A_{1}\right)= \frac{\prod_{i=1}^{K+1} m_{i}\left(A_{2}\right)}{\sum_{j=1}^{N} \prod_{i=1}^{K+1} m_{i}\left(A_{j}\right)} \\
& \cdots \ldots \ldots \\
& m^{k}\left(A_{1}\right)= \prod_{i=1}^{K+1} m_{i}\left(A_{N}\right) \\
& \sum_{j=1}^{N} \prod_{i=1}^{K+1} m_{i}\left(A_{j}\right)
\end{aligned}\right.
$$

They can be written as:

$$
m\left(A_{j}\right)=\frac{\prod_{i=1}^{K+1} m_{i}\left(A_{j}\right)}{\sum_{j=1}^{N} \prod_{i=1}^{k+1} m_{i}\left(A_{j}\right)},(j=1,2, \ldots N)
$$

Because the number of the evidence row is $n$, that is the maximum value of $K+1$ is $n$, hence the last combination results is:

$$
m\left(A_{j}\right)=\frac{\prod_{i=1}^{n} m_{i}\left(A_{j}\right)}{\sum_{j=1}^{N} \prod_{i=1}^{n} m_{i}\left(A_{j}\right)},(j=1,2, \ldots N)
$$

Up till now, we have completed the proof of the theorem 1.

Theorem 2: Provided a frame of discernment $X, A$ is a focal element of $X$. In $X$, there are $N$ nonvoid subsets of $A$ : $A_{1} A_{2, \ldots} \ldots A_{\mathrm{N}}$, these subsets do not overlap each other, and there are $n$ rows of independent evidence as (4). Suppose the evidence satisfy the normalization condition that

$$
\sum_{j=1}^{N} m_{i}\left(A_{j}\right)=1, i=1,2, \ldots n
$$

If the evidence of (4) are sequentially combined in pair by using the classical combination formulas of Dempster-Shafer evidence theory, the last combination results are written as $m\left(A_{1}\right), m\left(A_{2}\right) \ldots m\left(A_{N}\right)$.

Then the last combination results satisfy the normalization condition:

$\sum_{j=1}^{N} m\left(A_{j}\right)=1$

Proof: According to the last combined results in theorem 1, the sum of all BPAs of the last combined results is: 


$$
\begin{aligned}
\sum_{j=1}^{N} m\left(A_{j}\right)= & \frac{\prod_{i=1}^{n} m_{i}\left(A_{1}\right)}{\sum_{j=1}^{N} \prod_{i=1}^{n} m_{i}\left(A_{j}\right)}+\frac{\prod_{i=1}^{n} m_{i}\left(A_{2}\right)}{\sum_{j=1}^{N} \prod_{i=1}^{n} m_{i}\left(A_{j}\right)}+\ldots+\frac{\prod_{i=1}^{n} m_{i}\left(A_{N}\right)}{\sum_{j=1}^{N} \prod_{i=1}^{n} m_{i}\left(A_{j}\right)} \\
= & \frac{\prod_{i=1}^{n} m_{i}\left(A_{1}\right)+\prod_{i=1}^{n} m_{i}\left(A_{2}\right)+\ldots+\prod_{i=1}^{n} m_{i}\left(A_{N}\right)}{\sum_{j=1}^{N} \prod_{i=1}^{n} m_{i}\left(A_{j}\right)}=1
\end{aligned}
$$

Up till now, we have completed the proof of the theorem 2.

According to theorem 1 and theorem 2, we deduce two deductions as follows:

Deduction 1: Combine sequentially the evidence of (4) in pair using the combination formula of classical Dempster-Shafer evidence theory, the last combined results must be not related to the sequencing of the rows of the evidence.

Proof: Exchange the position of any two rows of evidence $m_{k}\left(A_{j}\right)$ and $m_{m}\left(A_{j}\right) \quad(j=1,2, . ., N)$ in (4), and let $k<m<n$, according to the last combined results in theorem 1, the combined results of foremost $k-1$ rows of evidence in (4) are as follows:

$$
m\left(A_{j}\right)=\frac{\prod_{i=1}^{k-1} m_{i}\left(A_{j}\right)}{\sum_{j=1}^{N} \prod_{i=1}^{k-1} m_{i}\left(A_{j}\right)},(j=1,2, \ldots N)
$$

The combined results of foremost $k$ rows of evidence in (4) are as follows:

$$
m\left(A_{j}\right)=\frac{m_{m}\left(A_{j}\right) \prod_{i=1}^{k-1} m_{i}\left(A_{j}\right)}{\sum_{j=1}^{N}\left[m_{m}\left(A_{j}\right) \prod_{i=1}^{k-1} m_{i}\left(A_{j}\right)\right]},(j=1,2, \ldots N)
$$

The combined results of foremost $m$ rows of evidence in (4) are as follows:

$$
m\left(A_{j}\right)=\frac{\prod_{i=1}^{m} m_{i}\left(A_{j}\right)}{\sum_{j=1}^{N} \prod_{i=1}^{m} m_{i}\left(A_{j}\right)},(j=1,2, \ldots N)
$$

The combined results of all $n$ rows of evidence in (4) are as follows:

$$
m\left(A_{j}\right)=\frac{\prod_{i=1}^{n} m_{i}\left(A_{j}\right)}{\sum_{j=1}^{N} \prod_{i=1}^{n} m_{i}\left(A_{j}\right)},(j=1,2, \ldots N)
$$

These results are as same as those which are obtained without exchange of the two rows of evidence. Up till now, we have completed the proof of the deduction 1.

Deduction 2: Combine sequentially the evidence of (4) in pair using the combination formula of classical Dempster-Shafer evidence theory, if there is at least one focal element's subset in which 
there is no any zero BPA component of the evidence in (8), it can be written as:

$$
m_{i}\left(A_{j}\right) \neq 0, i=1,2, \ldots, n ; j \in(1,2, \ldots N)
$$

then the last combined results must be convergent, that is the BPAs of the last result must concentrate on those subsets in which there is no any zero BPA component of evidence.

Proof: Suppose that there are $M$ evidence columns in which there are one zero BPA component of evidence at least in the $N$ evidence columns of (4). They can be written as:

$$
\begin{aligned}
& C_{1}=\left[m_{1}\left(A_{m_{1}}\right), m_{2}\left(A_{m_{1}}\right), \ldots ., m_{n}\left(A_{m_{1}}\right)\right]^{T}, 1 \leq m_{1} \leq N \\
& C_{2}=\left[m_{1}\left(A_{m_{2}}\right), m_{2}\left(A_{m_{2}}\right), \ldots ., m_{n}\left(A_{m_{2}}\right)\right]^{T}, 1 \leq m_{2} \leq N \\
& \ldots \ldots . . \\
& C_{M}=\left[m_{1}\left(A_{m_{M}}\right), m_{2}\left(A_{m_{M}}\right), \ldots ., m_{n}\left(A_{m_{M}}\right)\right]^{T}, 1 \leq m_{M} \leq N
\end{aligned}
$$

There is no zero BPA component of evidence in the rest $P$ evidence columns of (4), $P=N-M$. They can be written as:

$$
\begin{aligned}
& D_{1}=\left[m_{1}\left(A_{p_{1}}\right), m_{2}\left(A_{p_{1}}\right), \ldots ., m_{n}\left(A_{p_{1}}\right)\right]^{T}, 1 \leq p_{1} \leq N \\
& D_{2}=\left[m_{1}\left(A_{p_{2}}\right), m_{2}\left(A_{p_{2}}\right), \ldots ., m_{n}\left(A_{p_{2}}\right)\right]^{T}, 1 \leq p_{2} \leq N \\
& \ldots \ldots . . \\
& D_{P}=\left[m_{1}\left(A_{p_{P}}\right), m_{2}\left(A_{p_{P}}\right), \ldots ., m_{n}\left(A_{p_{P}}\right)\right]^{T}, 1 \leq p_{P} \leq N
\end{aligned}
$$

Then

$$
\begin{aligned}
& \prod_{i=1}^{n} m_{i}\left(A_{j}\right)=0, j=m_{1}, m_{2}, \ldots m_{M} \\
& \prod_{i=1}^{n} m_{i}\left(A_{j}\right) \neq 0, j=p_{1}, p_{2}, \ldots p_{P}
\end{aligned}
$$

According to the last combined results in theorem 1:

$$
\begin{gathered}
m\left(A_{j}\right)=\frac{\prod_{i=1}^{n} m_{i}\left(A_{j}\right)}{\sum_{j=1}^{N} \prod_{i=1}^{n} m_{i}\left(A_{j}\right)}=0,\left(j=m_{1}, m_{2}, \ldots m_{M}, M+P=N\right) \\
m\left(A_{j}\right)=\frac{\prod_{i=1}^{n} m_{i}\left(A_{j}\right)}{\sum_{j=1}^{N} \prod_{i=1}^{n} m_{i}\left(A_{j}\right)} \neq 0,\left(j=p_{1}, p_{2}, \ldots p_{P}, M+P=N\right)
\end{gathered}
$$

Therefore, the BPAs of the last result must concentrate on those focal elements in which there is no any zero BPA component of evidence. Up till now, we have completed the proof of deduction 2.

\section{Conclusion}

The results of research on theory and simulation show that, the step-by-step combination mixing the classic Dempster-Shafer method and the weighted average method can improve the combined result of the continuous substeps combining method further. Not only it can combine low-grade conflict evidence, but also can combine severe conflict evidence.Also this method has better convergence effectiveness and lesser uncertainty than the algorithms proposed in reference literature in combination of severe conflict evidence. 


\section{References}

[1] R.R. Yager, On the Dempster-Shafer framework and new combination rules, Information Sciences 41 (2) (1987) 93-137.

[2] Q.Sun, X.Q.Ye, W.K.Gu, A new combination rules of evidence theory, ACTA ELECTRONIC SINICA 28(8) (2000) 117-119.

[3] B.C. Li, B. Wang, J. Wei, An efficient combination rule of evidence theory, Journal of Data Acquisition \& Processing 17(1) (2002) 33-36 .

[4] Y. Deng, W. K. Shi, A modified combination rule of evidence theory, JOURNAL OF SHANGHAI JIAOTONG UNIVERSITY, 37(8) (2003) 1275-1278 .

[5] M. Z. Xiao, G. Y. Chen. Modified combination rule of evidence theory, Journal of Data Acquisition \& processing 19(4) (2004) 467-469 .

[6] Murphy C K. Combining belief functions when evidence conflicts[J]. Decision support systems, 2000, 29(1): 1-9.

[7] E. Lefevre, O. Colot, P. Vannoorenberghe, Belief function combination and the conflict management, Information Fusion 3 (2) (2002) 149-162.

[8] Ali T, Dutta P, Boruah H. A new combination rule for conflict problem of Dempster-Shafer evidence theory[J]. International Journal of Energy, Information and Communications, 2012, 3(1): 35-40.

[9] Dempster A P. Upper and lower probabilities induced by a multivalued mapping[J].The annals of mathematical statistics, 1967: 325-339. 